\title{
Dark Matter as the signal of Grand Unification
}

\author{
Mario Kadastik, ${ }^{1}$ Kristjan Kannike, ${ }^{1}$ and Martti Raidal ${ }^{1}$ \\ ${ }^{1}$ National Institute of Chemical Physics and Biophysics, Ravala 10, Tallinn 10143, Estonia
}

\begin{abstract}
We argue that the existence of Dark Matter (DM) is a possible consequence of GUT symmetry breaking. In GUTs like $S O(10)$, discrete $Z_{2}$ matter parity $(-1)^{3(B-L)}$ survives despite of broken $B-L$, and group theory uniquely determines that the only possible $Z_{2}$-odd matter multiplets belong to representation 16. We construct the minimal non-SUSY $S O(10)$ model containing one scalar 16 for DM and study its predictions below $M_{G}$. We find that EWSB occurs radiatively due to DM couplings to the SM Higgs boson. For thermal relic DM the mass range $M_{\mathrm{DM}} \sim \mathcal{O}(0.1-1) \mathrm{TeV}$ is predicted by model perturbativity up to $M_{G}$. For $M_{\mathrm{DM}} \sim \mathcal{O}(1) \mathrm{TeV}$ to explain the observed cosmic ray anomalies with DM decays, there exists a lower bound on the spin-independent direct detection cross section within the reach of planned experiments.
\end{abstract}

\section{INTRODUCTION}

The existence of Dark Matter (DM) of the Universe is now established without doubt 11. However, the fundamental physics behind it is unknown at present. In the most popular new physics scenario containing DM - supersymmetry - discrete $R$-parity is imposed by hand to prevent phenomenological disasters such as fast proton decay [2]. Similarly, in dedicated DM extensions of the standard model (SM) with new singlet [3], doublet [4] or higher multiplet scalars [5], ad hoc $Z_{2}$ symmetry must be added to ensure the stability of DM. These phenomenological models cannot answer the two most fundamental questions related to DM: $(i)$ why this particular multiplet or particle constitutes the DM of the Universe?; (ii) what is the origin of the imposed $Z_{2}$ symmetry? Therefore the underlying physics principles related to the existence of DM remain obscured.

In this work we argue that the existence of DM of the Universe can be a consequence of Grand Unification (GUT). The GUT framework not only explains the origin of DM but also determines the type of the DM particle and constrains its properties. In this scenario the existence of DM, non-zero neutrino masses via seesaw [6] and baryon asymmetry of the Universe via leptogenesis [7] all point to the same GUT framework.

We show that the $Z_{2}$ symmetry needed for DM stability could be a discrete remnant of GUT symmetry group, such as $S O(10)[8$ that we choose to work with in the following. When breaking $S O(10)$ down to the SM gauge group $S U(2)_{L} \times U(1)_{Y}$, the $S O(10)$ embedded $U(1)_{X}$, where $X$ is orthogonal to the SM hypercharge $Y$, leaves unbroken $Z_{2}$,9, 10 ,

$$
P_{X}=P_{M}=(-1)^{3(B-L)},
$$

which is the well known matter parity $P_{M}$. Due to its gauge origin $P_{M}$ is a symmetry of any SM extension including non-SUSY ones. In the latter case group theory predicts uniquely, without any detailed model building, that the only possible $Z_{2}$-odd multiplet under Eq. (1) is the 16 of $S O(10)$ [11. As inclusion of the fourth fermion generation $\mathbf{1 6}_{4}$ to the SM is not supported by experimental data, the non-SUSY $S O(10)$ GUT predicts that the DM is a mixture of $S U(2)_{L} \times U(1)_{Y} P_{M}$-odd complex scalar singlet $S$ and neutral component of doublet $H_{2}$ belonging to a new scalar 16 of $S O(10)$. Thus the DM of the Universe corresponds to the scalar analogues of the fermionic neutral matter fields, the right-handed neutrino $N_{R}$ and the left-handed neutrino $\nu_{L}$, respectively. Preserving $P_{M}$ requires $S O(10)$ breaking by an order parameter carrying even charge of $B-L$ [9, 10]. Therefore $S O(10)$ breaking also generates heavy Majorana masses which induce the seesaw mechanism as well as leptogenesis.

To test the proposed DM scenario we study the scalar potential of a minimal $S O(10)$ GUT model containing one scalar 16 for the DM and one scalar 10 for the SM Higgs doublet. We derive [12 renormalization group equations (RGEs) for scalar mass parameters $\mu_{i}^{2}$ and interaction couplings $\lambda_{i}$ below the GUT breaking scale and study the vacuum stability and perturbativity conditions for those parameters. We find that the SM Higgs mass parameter $\mu_{1}^{2}$ runs negative due to the presence of DM couplings with Higgs boson and triggers radiative electroweak symmetry breaking (EWSB) as in SUSY models [13]. Perturbativity up to $M_{G}=2 \times 10^{16} \mathrm{GeV}$ restricts all scalar self couplings to be $\lambda_{i}<1$ at $M_{Z}$ predicting a restricted mass window $70 \mathrm{GeV} \lesssim M_{\mathrm{DM}} \lesssim 2 \mathrm{TeV}$ for thermal relic DM mass. The operator $m /\left(\Lambda_{N} M_{P}\right) L L H_{1} H_{2}$ induces 3-body decays $D M \rightarrow l^{-} \nu W^{+}$which may explain the recently observed cosmic ray anomalies. For the DM mass preferred by this solution, $M_{\mathrm{DM}} \sim$ $\mathcal{O}(1) \mathrm{TeV}$, our framework predicts a lower bound on the spin independent direct cross section of DM with nuclei, which is within the reach of sensitivity of proposed experiments. 


\section{DM, LEPTOGENESIS AND SEESAW MECHANISM}

The $S O(10)$ gauge group contains two orthogonal $U(1)$ charges, which can be chosen to be the SM hypercharge $Y$ remaining unbroken after $S O(10)$ breaking at $M_{G}$, and broken $X=3(B-L)+4 T_{3 R}$, where $T_{3 R}$ is the third component of $S U(2)_{R} \in S O(10)$ isospin. If $S O(10)$ is broken by fields with even $X$ charge, the discrete subgroup $Z_{2}$ of $U(1)_{X}$ remains unbroken [9]. As $4 T_{3 R}$ is always even, the surviving $P_{X}$ parity is nothing but the matter parity, Eq. (1). Due to the $S O(10)$ breaking, $B-L$ is broken at GUT scale generating large Majorana masses for right-handed neutrinos $N_{R_{i}}$ which suppress the light neutrino masses via the seesaw mechanism. The $N_{R_{i}}$ decays in early Universe induce the baryon asymmetry via leptogenesis. Thus, in our model, the existence of DM due to the matter parity Eq. (1), the existence of baryon asymmetry and the existence of seesaw suppressed masses of light neutrinos have the same GUT origin. To our knowledge, $U(1)_{X}, X=5(B-L)-2 Y$ [14] has been used to forbid proton decay operators in GUTs, explicit examples of gauged $U(1)_{B-L}$ SUSY seesaw models generating $R$-parity have been presented in [15] and low energy non-SUSY SM extension with extra $U(1)^{\prime}$ gauge symmetry generating $Z_{2}$ is presented in [16], but the connection between non-supersymmetric DM and $P_{X}$ was first proposed in [11].

The $P_{X}$ parity of $S O(10)$ matter multiplets is uniquely determined by group theory. Therefore the proposed $S O(10)$ GUT scenario leaves no choice as to what are the DM particle multiplets. Under the group theoretic decomposition $S U(5) \times U(1)_{X}$ the $\mathbf{1 6}$ representation of $S O(10)$ reads $\mathbf{1 6}=\mathbf{1}^{16}(5)+\overline{\mathbf{5}}^{16}(-3)+\mathbf{1 0}^{16}(1)$, where the $X$ charges of the component multiplets are given in the brackets. While the $X$ charges are different, all the fields in 16 of $S O(10)$ are odd under the conserved $Z_{2}$ parity Eq. (1). Interestingly, fields in 16 provide the only $P_{X}$ odd particles because all other fields coming from small $S O(10)$ representations, 10, 45, 54, 120 and 126, are even under $P_{X}$. Thus the SM fermions belonging to $\mathbf{1 6}_{i}, i=1,2,3$, are all $P_{M}$-odd while the SM Higgs boson doublet is $P_{M}$-even because $\mathbf{1 0}=\mathbf{5}^{10}(-2)+\overline{\mathbf{5}}^{10}(2)$.

Although $B-L$ is broken in Nature by the heavy neutrino Majorana masses, discrete $(-1)^{3(B-L)}$ is respected by the interactions of all matter fields. Therefore, without any model building, general GUT group theoretic argument implies that the non-supersymmetric DM must belong to $\mathbf{1 6}$ of $S O(10)$. Adding a new fermionic $\mathbf{1 6}$ is equivalent to adding a new generation, which, due to mixing with lighter generations, cannot give DM. The only possibility is the new scalar 16 of $S O(10)$, which contains two DM candidates, the complex singlet $S$ and the neutral component of the doublet $\mathrm{H}_{2}$.

\section{MINIMAL SO(10) GUT INDUCED DM MODEL}

The $S O(10)$ symmetric scalar potential of one $\mathbf{1 6}$ and one $\mathbf{1 0 ,}$

$$
\begin{aligned}
V & =\mu_{1}^{2} \mathbf{1 0} \mathbf{1 0}+\lambda_{1}(\mathbf{1 0} \mathbf{1 0})^{2}+\mu_{2}^{2} \overline{\mathbf{1 6}} \mathbf{1 6}+\lambda_{2}(\overline{\mathbf{1 6}} \mathbf{1 6})^{2} \\
& +\lambda_{3}(\mathbf{1 0} \mathbf{1 0})(\overline{\mathbf{1 6}} \mathbf{1 6})+\lambda_{4}(\mathbf{1 6} \mathbf{1 0})(\overline{\mathbf{1 6}} \mathbf{1 0}) \\
& +\lambda_{S}^{\prime}\left[\mathbf{1 6}^{4}+\text { h.c. }\right]+\frac{\mu_{S H}^{\prime}}{2}[\mathbf{1 6} 1016+\text { h.c. }]
\end{aligned}
$$

provides the minimal example of GUT DM model. Here we have taken all parameters to be real for simplicity. We assume $S O(10)$ to break at $M_{G}$ down to $S U(2)_{L} \times U(1)_{Y} \times P_{M}$ in such a way that only one SM Higgs boson doublet $H_{1} \in \mathbf{1 0}$ and the DM candidate complex singlet $S \in \mathbf{1 6}$ and the inert doublet $H_{2} \in \mathbf{1 6}$ are light, with all other particle masses of order $M_{G}$. The $S O(10)$ symmetry breaking may occur in one or in several steps through intermediate symmetries such as $S U(5) \times U(1)_{X}$. We assume those steps to occur close to the GUT scale. Thus, between $M_{G}$ and the EWSB scale $M_{Z}$, the DM is described by the $H_{1} \rightarrow H_{1}, S \rightarrow-S, H_{2} \rightarrow-H_{2}$ 
invariant scalar potential

$$
\begin{aligned}
V & =\mu_{1}^{2} H_{1}^{\dagger} H_{1}+\lambda_{1}\left(H_{1}^{\dagger} H_{1}\right)^{2}+\mu_{2}^{2} H_{2}^{\dagger} H_{2}+\lambda_{2}\left(H_{2}^{\dagger} H_{2}\right)^{2} \\
& +\mu_{S}^{2} S^{\dagger} S+\frac{\mu_{S}^{\prime 2}}{2}\left[S^{2}+\left(S^{\dagger}\right)^{2}\right]+\lambda_{S}\left(S^{\dagger} S\right)^{2} \\
& +\frac{\lambda_{S}^{\prime}}{2}\left[S^{4}+\left(S^{\dagger}\right)^{4}\right]+\frac{\lambda_{S}^{\prime \prime}}{2}\left(S^{\dagger} S\right)\left[S^{2}+\left(S^{\dagger}\right)^{2}\right] \\
& +\lambda_{S 1}\left(S^{\dagger} S\right)\left(H_{1}^{\dagger} H_{1}\right)+\lambda_{S 2}\left(S^{\dagger} S\right)\left(H_{2}^{\dagger} H_{2}\right) \\
& +\frac{\lambda_{S 1}^{\prime}}{2}\left(H_{1}^{\dagger} H_{1}\right)\left[S^{2}+\left(S^{\dagger}\right)^{2}\right]+\frac{\lambda_{S 2}^{\prime}}{2}\left(H_{2}^{\dagger} H_{2}\right)\left[S^{2}+\left(S^{\dagger}\right)^{2}\right] \\
& +\lambda_{3}\left(H_{1}^{\dagger} H_{1}\right)\left(H_{2}^{\dagger} H_{2}\right)+\lambda_{4}\left(H_{1}^{\dagger} H_{2}\right)\left(H_{2}^{\dagger} H_{1}\right) \\
& +\frac{\lambda_{5}}{2}\left[\left(H_{1}^{\dagger} H_{2}\right)^{2}+\left(H_{2}^{\dagger} H_{1}\right)^{2}\right] \\
& +\frac{\mu_{S H}}{2}\left[S^{\dagger} H_{1}^{\dagger} H_{2}+\text { h.c. }\right]+\frac{\mu_{S H}^{\prime}}{2}\left[S H_{1}^{\dagger} H_{2}+\text { h.c. }\right]
\end{aligned}
$$

together with the GUT scale boundary conditions

$$
\begin{gathered}
\mu_{1}^{2}\left(M_{G}\right)>0, \mu_{2}^{2}\left(M_{G}\right)=\mu_{S}^{2}\left(M_{G}\right)>0 \\
\lambda_{2}\left(M_{G}\right)=\lambda_{S}\left(M_{G}\right)=\lambda_{S 2}\left(M_{G}\right), \lambda_{3}\left(M_{G}\right)=\lambda_{S 1}\left(M_{G}\right),
\end{gathered}
$$

and

$$
\begin{gathered}
\mu_{S}^{\prime 2}, \mu_{S H}^{2} \lesssim \mathcal{O}\left(\frac{M_{G}}{M_{P}}\right)^{n} \mu_{1,2}^{2} \\
\lambda_{5}, \lambda_{S 1}^{\prime}, \lambda_{S 2}^{\prime}, \lambda_{S}^{\prime \prime} \lesssim \mathcal{O}\left(\frac{M_{G}}{M_{P}}\right)^{n} \lambda_{1,2,3,4} .
\end{gathered}
$$

We require $\mu_{i}^{2}\left(M_{G}\right)>0$ in order not to break the SM gauge symmetry spontaneously at GUT scale. While the parameters in Eq. (4) are allowed by $S O(10)$, the ones in Eq. (5) can be generated only after $S O(10)$ breaking by operators suppressed by $n$ power of Planck scale $M_{P}$. If all parameters in Eq. (5) vanished identically, PecceiQuinn (PQ) symmetry would imply degenerate real and imaginary components of DM. However, direct search for inelastic DM requires the mass splitting to exceed $\mathcal{O}(100) \mathrm{keV}$. Smallness of $\lambda_{5}$, as given by Eq. (5), allows one to interpret the annual modulation observed by DAMA experiment with inelastic scattering of DM in the Inert Doublet Model [17. In our model there are more possibilities to obtain small mass splitting between real and imaginary components of DM candidates, $c f$. Eq. (3). In the following we assume the PQ symmetry to be broken softly by $0<\left|\mu_{S}^{\prime 2}\right| \ll\left|\mu_{1}^{2}\right|$.

\section{VACUUM STABILITY CONSTRAINTS}

In the SM the requirements of vacuum stability and scalar potential perturbativity up to $M_{G}$ put the lower and upper bounds on the Higgs boson mass $127 \mathrm{GeV}<M_{H}<170 \mathrm{GeV}$, respectively (see [18] and references therein). In our model the vacuum stability requires

$$
\begin{aligned}
\lambda_{1} & >0, & \lambda_{3} & >-2 \sqrt{\lambda_{1} \lambda_{2}}, \\
\lambda_{2} & >0, & \lambda_{3}+\lambda_{4}-\left|\lambda_{5}\right| & >-2 \sqrt{\lambda_{1} \lambda_{2}}, \\
\lambda_{S}+\lambda_{S}^{\prime} & >\left|\lambda_{S}^{\prime \prime}\right|, & 8\left(\lambda_{S}-\lambda_{S}^{\prime}\right) \lambda_{S}^{\prime} & >\lambda_{S}^{\prime \prime 2}, \\
4 \lambda_{1}\left(\lambda_{S}+\lambda_{S}^{\prime}+\lambda_{S}^{\prime \prime}\right) & >\left(\lambda_{S 1}+\lambda_{S 1}^{\prime}\right)^{2}, & 4 \lambda_{2}\left(\lambda_{S}+\lambda_{S}^{\prime}+\lambda_{S}^{\prime \prime}\right) & >\left(\lambda_{S 2}+\lambda_{S 2}^{\prime}\right)^{2}, \\
4 \lambda_{1}\left(\lambda_{S}+\lambda_{S}^{\prime}-\lambda_{S}^{\prime \prime}\right) & >\left(\lambda_{S 1}-\lambda_{S 1}^{\prime}\right)^{2}, & 4 \lambda_{2}\left(\lambda_{S}+\lambda_{S}^{\prime}-\lambda_{S}^{\prime \prime}\right) & >\left(\lambda_{S 2}-\lambda_{S 2}^{\prime}\right)^{2} .
\end{aligned}
$$

Because there are more scalar couplings than in the SM, they can counteract the top quark Yukawa term in the beta function for $\lambda_{1}$ presented in the next Section and lower the vacuum stability bound on $M_{H}$ below the LEP2 experimental bound of $114.4 \mathrm{GeV}$ consistently with Eq. (6). Therefore, in our model, the precision data indications for light SM Higgs boson does not contradict with vacuum stability constraints. 


\section{RGE ANALYSES}

Because our low energy model is induced by GUT, it must stay perturbative up to the GUT scale. This requirement implies stringent constraints on the model parameters and consequently on the properties of DM. We have derived [12] the full set of RGEs of the model Eq. (3). The one-loop beta functions are given by

$$
\begin{aligned}
& \beta_{\lambda_{1}}=24 \lambda_{1}^{2}+2 \lambda_{3}^{2}+2 \lambda_{3} \lambda_{4}+\lambda_{4}^{2}+\lambda_{5}^{2}+\lambda_{S 1}^{2}+\lambda_{S 1}^{\prime 2} \\
& +\frac{3}{8}\left(3 g^{4}+g^{4}+2 g^{2} g^{2}\right)-3 \lambda_{1}\left(3 g^{2}+g^{\prime 2}-4 y_{t}^{2}\right)-6 y_{t}^{4}, \\
& \beta_{\lambda_{2}}=24 \lambda_{2}^{2}+2 \lambda_{3}^{2}+2 \lambda_{3} \lambda_{4}+\lambda_{4}^{2}+\lambda_{5}^{2}+\lambda_{S 2}^{2}+\lambda_{S 2}^{\prime 2} \\
& +\frac{3}{8}\left(3 g^{4}+g^{\prime 4}+2 g^{2} g^{\prime 2}\right)-3 \lambda_{2}\left(3 g^{2}+g^{\prime 2}\right) \\
& \beta_{\lambda_{3}}=4\left(\lambda_{1}+\lambda_{2}\right)\left(3 \lambda_{3}+\lambda_{4}\right)+4 \lambda_{3}^{2}+2 \lambda_{4}^{2}+2 \lambda_{5}^{2}+2 \lambda_{S 1} \lambda_{S 2}+2 \lambda_{S 1}^{\prime} \lambda_{S 2}^{\prime} \\
& +\frac{3}{4}\left(3 g^{4}+g^{4}-2 g^{2} g^{2}\right)-3 \lambda_{3}\left(3 g^{2}+g^{\prime 2}-2 y_{t}^{2}\right), \\
& \beta_{\lambda_{4}}=4\left(\lambda_{1}+\lambda_{2}\right) \lambda_{4}+8 \lambda_{3} \lambda_{4}+4 \lambda_{4}^{2}+8 \lambda_{5}^{2} \\
& +3 g^{2} g^{\prime 2}-3 \lambda_{4}\left(3 g^{2}+g^{\prime 2}-2 y_{t}^{2}\right) \\
& \beta_{\lambda_{5}}=4\left(\lambda_{1}+\lambda_{2}+2 \lambda_{3}+3 \lambda_{4}\right) \lambda_{5} \\
& -3 \lambda_{5}\left(3 g^{2}+g^{\prime 2}-2 y_{t}^{2}\right) \\
& \beta_{\lambda_{S}}=20 \lambda_{S}^{2}+2 \lambda_{S 1}^{2}+\lambda_{S 1}^{\prime 2}+2 \lambda_{S 2}^{2}+\lambda_{S 2}^{\prime 2}+36 \lambda_{S}^{\prime 2}+\frac{27}{2} \lambda_{S}^{\prime \prime 2}, \\
& \beta_{\lambda_{S}^{\prime}}=\lambda_{S 1}^{\prime 2}+\lambda_{S 2}^{\prime 2}+24 \lambda_{S} \lambda_{S}^{\prime}+\frac{9}{2} \lambda_{S}^{\prime \prime 2} \\
& \beta_{\lambda_{S}^{\prime \prime}}=4 \lambda_{S 1} \lambda_{S 1}^{\prime}+4 \lambda_{S 2} \lambda_{S 2}^{\prime}+36\left(\lambda_{S}+\lambda_{S}^{\prime}\right) \lambda_{S}^{\prime \prime}, \\
& \beta_{\lambda_{S 1}}=4\left(3 \lambda_{1}+2 \lambda_{S}+\lambda_{S 1}\right) \lambda_{S 1}+4 \lambda_{S 1}^{\prime 2}+\left(4 \lambda_{3}+2 \lambda_{4}\right) \lambda_{S 2}+6 \lambda_{S 1}^{\prime} \lambda_{S}^{\prime \prime} \\
& -\frac{3}{2}\left(3 g^{2}+g^{\prime 2}-4 y_{t}^{2}\right) \lambda_{S 1} \\
& \beta_{\lambda_{S 2}}=4\left(3 \lambda_{2}+2 \lambda_{S}+\lambda_{S 2}\right) \lambda_{S 2}+4 \lambda_{S 2}^{\prime 2}+\left(4 \lambda_{3}+2 \lambda_{4}\right) \lambda_{S 1}+6 \lambda_{S 2}^{\prime} \lambda_{S}^{\prime \prime} \\
& -\frac{3}{2}\left(3 g^{2}+g^{2}\right) \lambda_{S 2} \\
& \beta_{\lambda_{S 1}^{\prime}}=\left(4 \lambda_{3}+2 \lambda_{4}\right) \lambda_{S 2}^{\prime}+4\left(3 \lambda_{1}+\lambda_{S}+2 \lambda_{S 1}+3 \lambda_{S}^{\prime}\right) \lambda_{S 1}^{\prime}+6 \lambda_{S 1} \lambda_{S}^{\prime \prime} \\
& -\frac{3}{2}\left(3 g^{2}+g^{\prime 2}-4 y_{t}^{2}\right) \lambda_{S 1}^{\prime} \\
& \beta_{\lambda_{S 2}^{\prime}}=\left(4 \lambda_{3}+2 \lambda_{4}\right) \lambda_{S 1}^{\prime}+4\left(3 \lambda_{2}+\lambda_{S}+2 \lambda_{S 2}+3 \lambda_{S}^{\prime}\right) \lambda_{S 2}^{\prime}+6 \lambda_{S 2} \lambda_{S}^{\prime \prime} \\
& -\frac{3}{2}\left(3 g^{2}+g^{\prime 2}\right) \lambda_{S 2}^{\prime} \\
& \beta_{\mu_{1}^{2}}=12 \mu_{1}^{2} \lambda_{1}+4 \mu_{2}^{2} \lambda_{3}+2 \mu_{2}^{2} \lambda_{4}+2 \mu_{S}^{2} \lambda_{S 1}+2 \mu_{S}^{\prime 2} \lambda_{S 1}^{\prime}+\frac{1}{2}\left(\mu_{S H}^{2}+\mu_{S H}^{\prime 2}\right) \\
& -\frac{3}{2} \mu_{1}^{2}\left(3 g^{2}+g^{\prime 2}-4 y_{t}^{2}\right) \\
& \beta_{\mu_{2}^{2}}=12 \mu_{2}^{2} \lambda_{2}+4 \mu_{1}^{2} \lambda_{3}+2 \mu_{1}^{2} \lambda_{4}+2 \mu_{S}^{2} \lambda_{S 2}+2 \mu_{S}^{\prime 2} \lambda_{S 2}^{\prime}+\frac{1}{2}\left(\mu_{S H}^{2}+\mu_{S H}^{\prime 2}\right) \\
& -\frac{3}{2} \mu_{2}^{2}\left(3 g^{2}+g^{\prime 2}\right) \\
& \beta_{\mu_{S}^{2}}=8 \mu_{S}^{2} \lambda_{S}+4 \mu_{1}^{2} \lambda_{S 1}+4 \mu_{2}^{2} \lambda_{S 2}+6 \mu_{S}^{\prime 2} \lambda_{S}^{\prime \prime}+\mu_{S H}^{2}+\mu_{S H}^{\prime 2}, \\
& \beta_{\mu_{S}^{\prime 2}}=4 \mu_{S}^{\prime 2} \lambda_{S}+4 \mu_{1}^{2} \lambda_{S 1}^{\prime}+4 \mu_{2}^{2} \lambda_{S 2}^{\prime}+12 \mu_{S}^{\prime 2} \lambda_{S}^{\prime}+6 \mu_{S}^{2} \lambda_{S}^{\prime \prime}+2 \mu_{S H} \mu_{S H}^{\prime} \text {, } \\
& \beta_{\mu_{S H}}=2 \mu_{S H}\left(\lambda_{3}+2 \lambda_{4}+\lambda_{S 1}+\lambda_{S 2}\right)+2 \mu_{S H}^{\prime}\left(3 \lambda_{5}+\lambda_{S 1}^{\prime}+\lambda_{S 2}^{\prime}\right) \\
& -\frac{3}{2} \mu_{S H}\left(3 g^{2}+g^{\prime 2}-2 y_{t}^{2}\right) \\
& \beta_{\mu_{S H}^{\prime}}=2 \mu_{S H}^{\prime}\left(\lambda_{3}+2 \lambda_{4}+\lambda_{S 1}+\lambda_{S 2}\right)+2 \mu_{S H}\left(3 \lambda_{5}+\lambda_{S 1}^{\prime}+\lambda_{S 2}^{\prime}\right) \\
& -\frac{3}{2} \mu_{S H}^{\prime}\left(3 g^{2}+g^{2}-2 y_{t}^{2}\right) \text {. }
\end{aligned}
$$




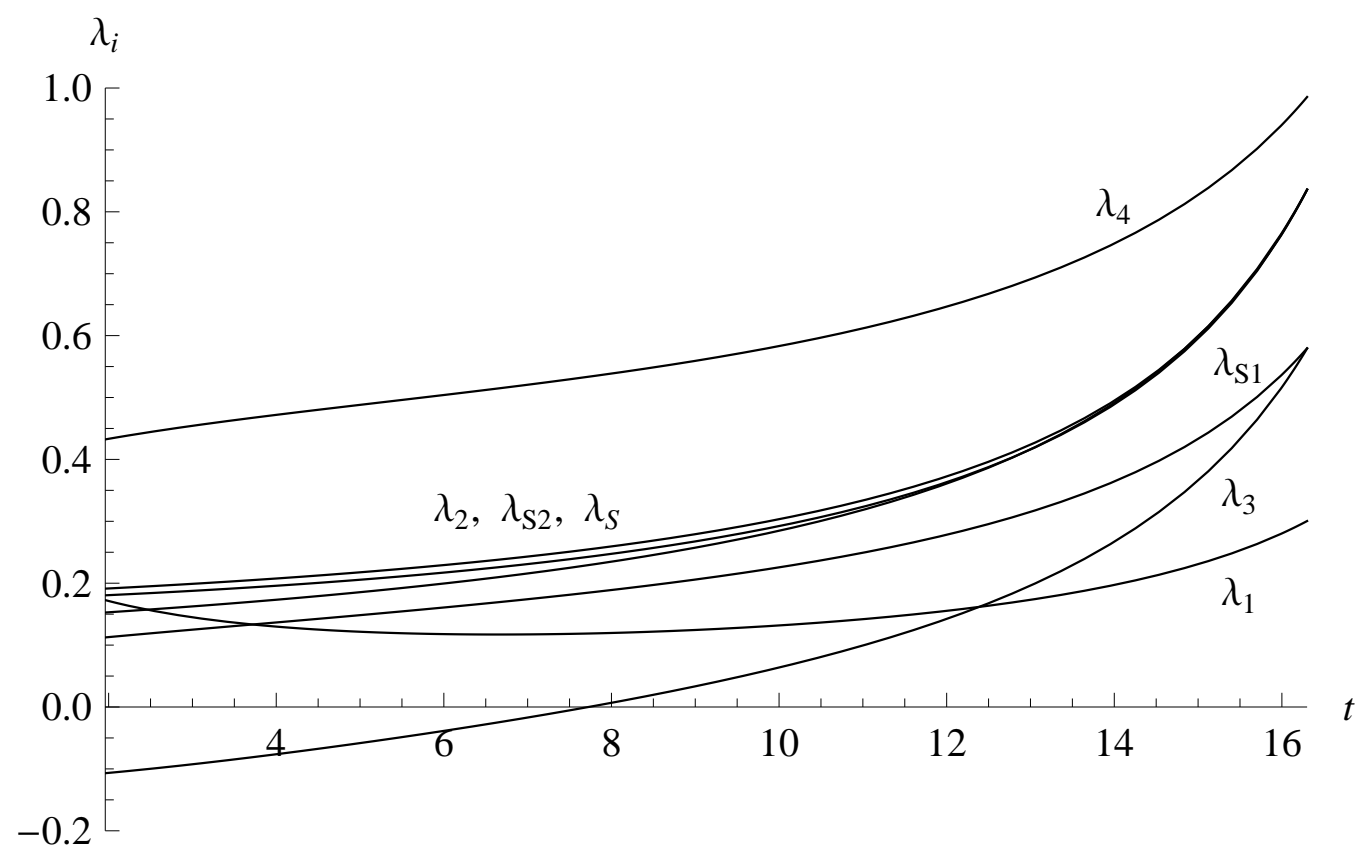

Figure 1: An example of $\lambda_{i}$ running from $M_{G}$ to $M_{Z}$. All $\lambda_{i}$ not suppressed by $S O(10)$ boundary conditions Eq. (5) are shown.

We also include the one-loop $\beta$-functions for $g, g^{\prime}, g_{3}$ and $y_{t}$, given by

$$
\begin{aligned}
\beta_{g^{\prime}} & =7 g^{\prime 3}, \\
\beta_{g} & =-3 g^{3}, \\
\beta_{g_{3}} & =-7 g^{3}, \\
\beta_{y_{t}} & =y_{t}\left(\frac{9}{2} y_{t}^{2}-\frac{17}{12} g^{\prime 2}-\frac{9}{4} g^{2}-8 g_{3}^{2}\right) .
\end{aligned}
$$

Based solely on the running due to the low-energy RGEs, we identify the unification scale $2 \times 10^{16} \mathrm{GeV}$ by the RGE solution for $g_{2}=g_{3}$. The exact values of gauge couplings at $M_{G}$ are given by $g_{1}=\sqrt{5 / 3} g^{\prime}=0.58$, $g_{2}=g_{3}=0.53$. Based solely on the running due to the low-energy RGEs, the precision of unification of all three gauge couplings in our model is better than in the SM because of the existence of an extra scalar doublet. We assume that an exact unification can be achieved due to the GUT thresholds corrections in full $S O(10)$ theory which we cannot estimate because the details of GUT symmetry breaking are not known. Those corrections can have only small logarithmic influence on our numerical estimates of $g_{i}$ just below $M_{G}$ and affect our numerical results for DM negligibly. In our numerical analysis we follow the strategy used in similar studies of parameter running in SUSY GUT theories. We fix all the measured model parameters and the SM Higgs boson mass at $M_{Z}$ and calculate the corresponding $\mu_{1}^{2}$ and $\lambda_{1}$. We run them up to the GUT scale where we randomly generate new physics parameters assuming the $S O(10)$ boundary conditions. We iterate running until the relative error between fixed and calculated $\mu_{1}^{2}$ and $\lambda_{1}$ at $M_{Z}$ gets smaller than 1\%. After that we calculate the DM abundance and direct detection cross section at $M_{Z}$.

We find that the new physics parameters are strongly constrained by the vacuum stability and perturbativity arguments. For example, assuming all $\lambda_{i}$ allowed by Eq. (2) to be equal at $M_{Z}$, perturbativity of them up to $M_{G}$ requires $\lambda_{i}\left(M_{Z}\right)<0.194,0.187,0.170$ for $M_{H}=120,140,160 \mathrm{GeV}$, respectively. We also impose the GUT boundary conditions Eq. (4).

We present one consistent example of the evolution of scalar self couplings $\lambda_{i}$ and mass parameters $\mu_{i}$ between $M_{Z}$ and $M_{G}$ in Fig. 1 and in Fig. 2 , respectively. We assume $M_{H}=140 \mathrm{GeV}$ and take $\mu_{S H}^{\prime}\left(M_{G}\right)=1 \mathrm{GeV}$. The couplings $\lambda_{i}\left(M_{Z}\right)$ must be small as not to reach the Landau pole below $M_{G}$. The SM gauge symmetry $S U(2)_{L} \times U(1)_{Y}$ is not broken at $M_{G}$ because all scalar mass parameters are positive $\mu_{i}^{2}\left(M_{G}\right)>0$. However, the SM Higgs mass parameter $\mu_{1}^{2}$ exhibits stronger running than the DM mass parameters and triggers the radiative EWSB as in SUSY models 13. In our case the EWSB is induced by DM couplings to the SM Higgs boson. (Previously, EWSB via a Coleman-Weinberg-like mechanism has been considered in the Inert Doublet Model [19].) 


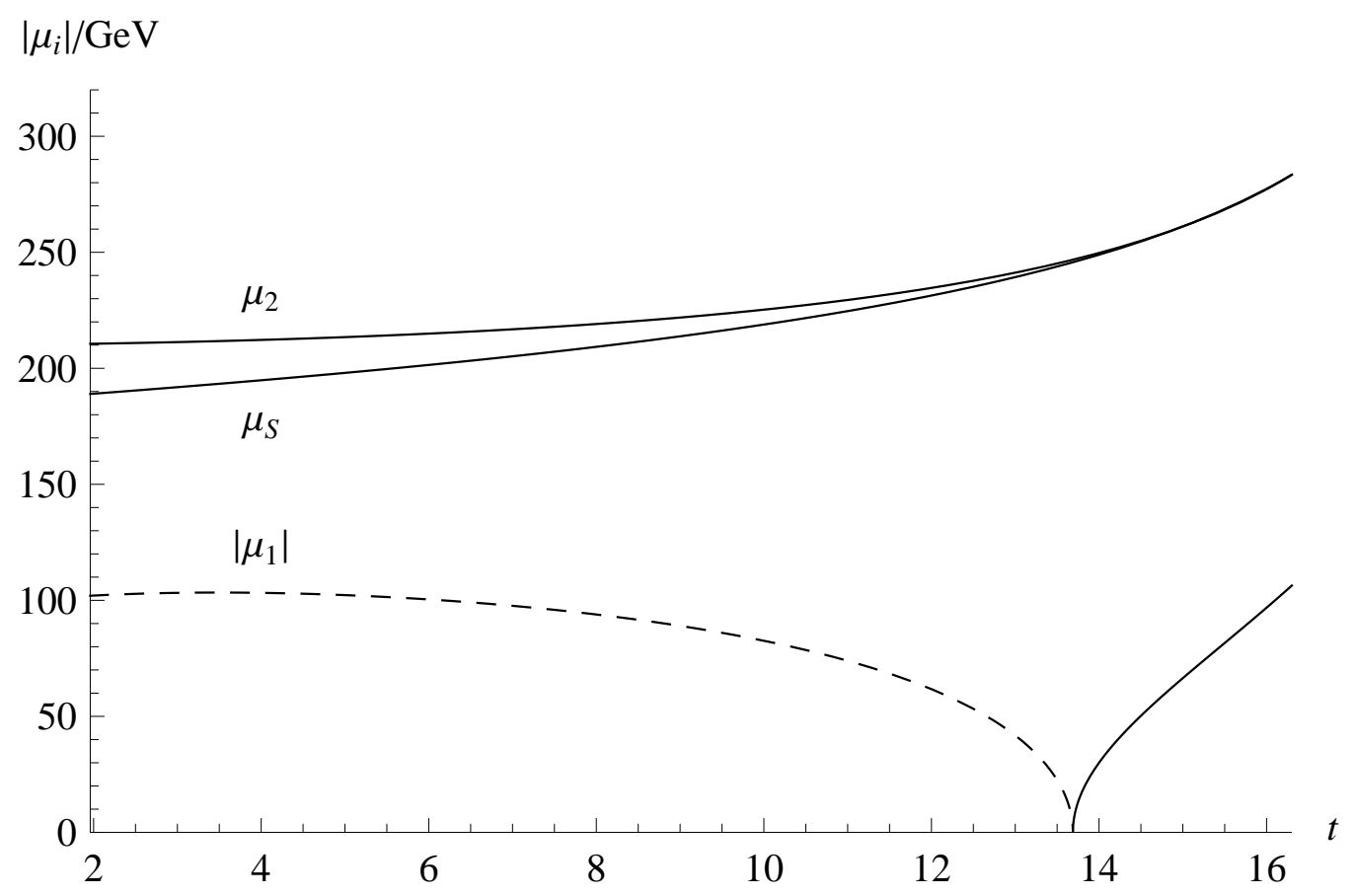

Figure 2: An example of $\mu_{1,2, S}$ running from $M_{G}$ to $M_{Z}$. Dashed line represents negative values of $\mu_{1}^{2}$ inducing EWSB.

An interesting feature of the model, demonstrated in Fig. 2, is that the singlet DM mass parameter at low energies is always smaller than the doublet one, $\mu_{S}^{2}\left(M_{Z}\right)<\mu_{2}^{2}\left(M_{Z}\right)$. Thus, for small singlet-doublet mixing as is assumed in this example, the DM particle is predominantly scalar singlet $S$ whose real and imaginary component mass degeneracy is lifted by small $\left|\mu_{S}^{\prime 2}\right| \ll\left|\mu_{1}^{2}\right|$.

\section{PREDICTIONS FOR DM MASS AND DIRECT DETECTION CROSS SECTION}

We assume that DM is a thermal relic and calculate its abundance and direct cross section with matter using the MicrOMEGAs package [20. The DM interactions (3) were calculated using the FeynRules package [21]. We scan over the entire parameter space satisfying Eq. (4), and calculate the RGE evolution of those parameters down to the EW scale.

Fig. 3 presents a scattered plot of the spin-independent DM direct detection cross section per nucleon as a function of DM mass $M_{\mathrm{DM}}$ for the SM Higgs boson mass range from $115 \mathrm{GeV}$ (red) to $170 \mathrm{GeV}$ (violet). The whole parameter space allowed by theoretical constraints of vacuum stability and positive masses and experimental constraints from LEP2 and the WMAP $3 \sigma$ result $0.094<\Omega_{D M} h^{2}<0.129$ [ $]$ is shown. After fixing $\lambda_{1}$ and $\mu_{1}^{2}$ from the assumed SM Higgs boson mass, we randomly generate the remaining scalar self couplings and mass parameters at $M_{G}$ in the ranges $0<\left|\lambda_{i}\right|<4 \pi$ and $0<\mu_{i}^{2}<(10 \mathrm{TeV})^{2}$. After RGE running the numerical ranges for non-zero parameters at $M_{Z}$ are $0.117<\lambda_{1}<0.239,0.024<\lambda_{2}<0.227,-0.424<\lambda_{3}<0.247$, $-0.584<\lambda_{4}<0.599,0.037<\lambda_{S}<0.177,0.000<\lambda_{S}^{\prime}<0.098,-0.212<\lambda_{S 1}<0.221,0.031<\lambda_{S 2}<0.234$, $-14548 \mathrm{GeV}^{2}<\mu_{1}^{2}<-7056 \mathrm{GeV}^{2}, 3147 \mathrm{GeV}^{2}<\mu_{2}^{2}<1.72 \times 10^{7} \mathrm{GeV}^{2}, 2894 \mathrm{GeV}^{2}<\mu_{S}^{2}<3.84 \times 10^{6} \mathrm{GeV}^{2}$, $-11634 \mathrm{GeV}<\mu_{S H}^{\prime}<11504 \mathrm{GeV}$.

The present 22] and future [23] experimental sensitivities for DM direct detection are shown in Fig. 3. As a result, we find that the DM mass is restricted to the window $70 \mathrm{GeV} \lesssim M_{\mathrm{DM}} \lesssim 2 \mathrm{TeV}$. The lower bound comes from non-observation of charged scalars at LEP2. The upper bound $M_{\mathrm{DM}} \lesssim 2 \mathrm{TeV}$ comes from the the requirement of perturbativity of the model parameters up to $M_{G}$. Therefore, the DM mass scale $M_{\mathrm{DM}} \lesssim \mathcal{O}(0.1-$ 1) $\mathrm{TeV}$ is a prediction of our scalar DM GUT scenario.

The direct DM interaction with nuclei occurs via the SM Higgs boson exchange. The dominant DM-Higgs effective coupling involved in this process is

$$
\lambda_{\text {eff }} v=\frac{1}{2}\left(\sqrt{2} s c \mu_{S H}^{\prime}+2 s^{2}\left(\lambda_{3}+\lambda_{4}\right) v+2 c^{2} \lambda_{S 1} v\right)
$$




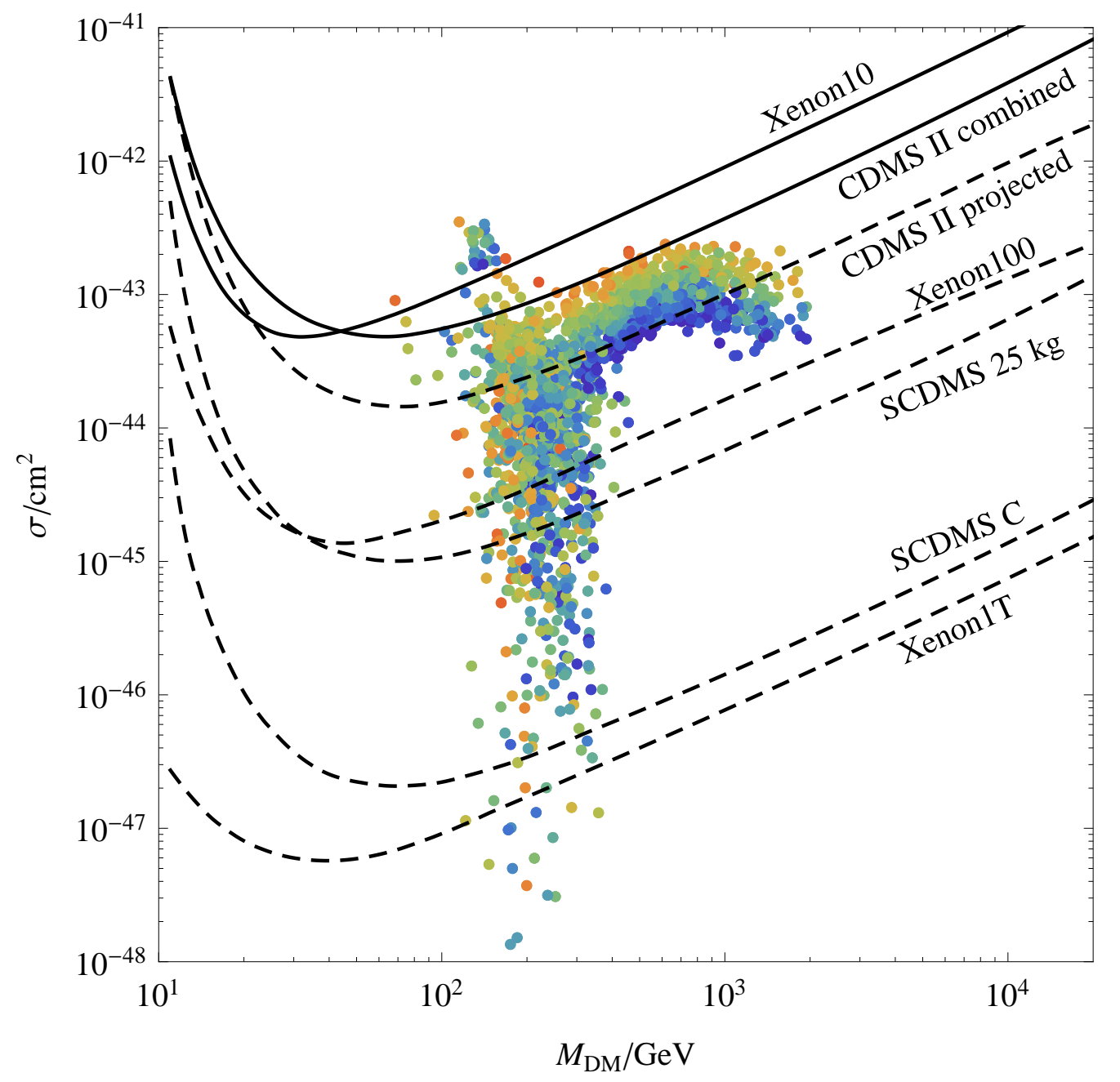

Figure 3: DM direct detection cross-section per nucleon vs. $M_{\mathrm{DM}}$. Color shows SM Higgs masses from $115 \mathrm{GeV}$ (red) to $170 \mathrm{GeV}$ (violet). The points shown encompass the whole parameter space allowed by theoretical and experimental constraints.

where $s, c$ are the sine, cosine of the singlet-doublet mixing angle. If $M_{\mathrm{DM}} \lesssim 300 \mathrm{GeV}$, cancellation between different terms in Eq. (9) is possible and the spin independent direct detection cross section can be accidentally small, $c f$. Fig. 3. However, for larger DM masses both Eq. (9) and thermal freeze-out cross section are dominated by large $\mu_{S H}^{\prime}$ term and one obtains a relation between the DM abundance and the direct detection cross sections with only mild dependence on $M_{H}$ via RGEs. For $M_{\mathrm{DM}}=1 \mathrm{TeV}$ the WMAP result predicts a lower bound $\sigma / n>2 \cdot 10^{-43}\left(115 \mathrm{GeV} / M_{H}\right)^{4} \mathrm{~cm}^{2}$ which is well within the reach of the planned experiments, $c f$. Fig. 3 . Should CDMS experiment observe DM scattering on nuclei, light SM Higgs boson mass is indicated in agreement with precision electroweak data.

\section{DM INDIRECT DETECTION}

The PAMELA [24, ATIC [25], HESS [26] and Fermi [27] anomalies of cosmic ray positron/electron fluxes can be explained with $\mathcal{O}(1) \mathrm{TeV}$ mass DM decays [28] via $d=6$ operators [29], preferably to multi-particle final state [30, 31. Non-observation of photons associated with DM annihilation in the Galactic center [32] and in DM haloes in the Universe [33] as well as the suppression of hadronic annihilation modes [34] strongly favor DM decays over annihilations as a solution to the anomalies.

In our scenario the decays of DM are most naturally explained via the seesaw like operator $L L H_{1} H_{2}$ which, in addition to the suppression by heavy Majorana neutrino scale $M_{N}$, must be suppressed by the $Z_{2}$ breaking 
effects by additional $M_{P}$. We obtain that below EWSB scale the dominant decay mode is given by

$$
\frac{\lambda_{N}}{M_{N}} \frac{m}{M_{P}} L L H_{1} H_{2} \rightarrow 10^{-30} \mathrm{GeV}^{-1} \nu l^{-} W^{+} H_{2}^{0},
$$

where we have taken $\lambda_{N} \sim 1, M_{N} \sim 10^{14} \mathrm{GeV}$ and $m \sim v \sim 100 \mathrm{GeV}$. In the decays of $W^{+}$antiprotons are produced about in $10 \%$ of decays. Such a small fraction of antiprotons is till allowed by PAMELA data taking into account uncertainties in the cosmic ray propagation models [35. Such a small effective Yukawa coupling of Eq. 10 can explain the long DM lifetime $10^{26} \mathrm{~s}$ without conflicting with the present observational constraints.

\section{CONCLUSIONS}

We have argued that the existence of DM, the baryon asymmetry of the Universe, and small neutrino masses may all signal the same underlying GUT physics. Although $B-L$ is broken in Nature by heavy neutrino Majorana masses, $Z_{2}$ parity $(-1)^{3(B-L)}$ is respected by interactions of all matter fields. Hence, group theory predicts that in $S O(10)$ GUTs the non-supersymmetric DM must be contained in the scalar representation 16.

Based on $S O(10)$ GUT, we have presented a minimal DM model, calculated the full set of its RGEs and studied its predictions. We find that the EWSB occurs radiatively due to SM Higgs boson couplings to the DM, analogously to SUSY models. The thermal relic DM mass is predicted to be $M_{\mathrm{DM}} \lesssim \mathcal{O}(0.1-1) \mathrm{TeV}$ by the requirement of perturbativity of model parameters up to the GUT scale. If $M_{\mathrm{DM}} \gtrsim 300 \mathrm{GeV}$ as suggested by DM decay solution to the recently observed cosmic ray anomalies, the WMAP measurement of DM abundance predicts a lower bound on DM spin independent direct cross section with nuclei, which is within the reach of planned experiments for all values of the SM Higgs boson mass.

Acknowledgment. We thank M. Tytgat for communication. This work was supported by the ESF Grant 8090 and by EU FP7-INFRA-2007-1.2.3 contract No 223807.

[1] E. Komatsu et al. [WMAP Collaboration], Astrophys. J. Suppl. 180, 330 (2009), arXiv:0803.0547.

[2] G. R. Farrar and P. Fayet, Phys. Lett. B 76, 575 (1978); S. Dimopoulos and H. Georgi, Nucl. Phys. B 193, 150 (1981); L. Ibanez and G. Ross, Nucl. Phys. B 368, 3 (1992).

[3] J. McDonald, Phys. Rev. D 50, 3637 (1994); C. P. Burgess, M. Pospelov and T. ter Veldhuis, Nucl. Phys. B 619, 709 (2001); V. Barger et al., Phys. Rev. D 77, 035005 (2008), Phys. Rev. D 79, 015018 (2009).

[4] N. G. Deshpande and E. Ma, Phys. Rev. D 18, 2574 (1978); E. Ma, Phys. Rev. D 73, 077301 (2006); R. Barbieri, L. J. Hall and V. S. Rychkov, Phys. Rev. D 74, 015007 (2006), arXiv:hep-ph/0603188 L. Lopez Honorez, E. Nezri, J. F. Oliver and M. H. G. Tytgat, JCAP 0702, 028 (2007) arXiv:hep-ph/0612275.

[5] T. Hambye, F. S. Ling, L. Lopez Honorez and J. Rocher, JHEP 0907, 090 (2009) arXiv:0903.4010 [hep-ph]].

[6] P. Minkowski, Phys. Lett. B 67, 421 (1977); T. Yanagida, in Baryon Number of the Universe and Unified Theories, Tsukuba, Japan, 13-14 Feb 1979; M. Gell-Mann, P. Ramond and R. Slansky, in Supergravity, P. van Nieuwenhuizen and D.Z. Freedman (eds.), North Holland Publ. Co., 1979; S. L. Glashow, NATO Adv. Study Inst. Ser. B Phys. 59 (1979) 687; R. N. Mohapatra and G. Senjanovic, Phys. Rev. Lett. 44 (1980) 912.

[7] M. Fukugita and T. Yanagida, Phys. Lett. B 174, 45 (1986).

[8] H. Fritzsch and P. Minkowski, Annals Phys. 93, 193 (1975).

[9] L. M. Krauss and F. Wilczek, Phys. Rev. Lett. 62, 1221 (1989).

[10] S. P. Martin, Phys. Rev. D 46, 2769 (1992).

[11] M. Kadastik, K. Kannike and M. Raidal, arXiv:0903.2475.

[12] C. Ford, I. Jack and D. R. T. Jones, Nucl. Phys. B 387, 373 (1992) [Erratum-ibid. B 504, 551 (1997)]; P. M. Ferreira and D. R. T. Jones, arXiv:0903.2856.

[13] L. E. Ibanez and G. G. Ross, Phys. Lett. B 110, 215 (1982).

[14] F. Wilczek and A. Zee, Phys. Lett. B 88, 311 (1979); N. Sakai and T. Yanagida, Nucl. Phys. B 197, 533 (1982).

[15] S. P. Martin, Phys. Rev. D 54, 2340 (1996).

[16] J. Kubo and D. Suematsu, Phys. Lett. B 643, 336 (2006) arXiv:hep-ph/0610006; D. Suematsu, Eur. Phys. J. C 56, 379 (2008) arXiv:0706.2401 [hep-ph]].

[17] C. Arina, F. S. Ling and M. H. G. Tytgat, arXiv:0907.0430 [hep-ph].

[18] M. Sher, Phys. Rept. 179, 273 (1989). P. M. Ferreira and D. R. T. Jones, arXiv:0903.2856.

[19] T. Hambye and M. H. G. Tytgat, Phys. Lett. B 659, 651 (2008) arXiv:0707.0633 [hep-ph]].

[20] G. Belanger, F. Boudjema, A. Pukhov and A. Semenov, Comput. Phys. Commun. 176 (2007) 367; G. Belanger, F. Boudjema, A. Pukhov and A. Semenov, Comput. Phys. Commun. 180, 747 (2009), arXiv:0803.2360.

[21] N. D. Christensen and C. Duhr, arXiv:0806.4194 
[22] Z. Ahmed et al. [CDMS Collaboration], Phys. Rev. Lett. 102, 011301 (2009), arXiv:0802.3530, G. J. Alner et al., Astropart. Phys. 28, 287 (2007), arXiv:astro-ph/0701858.

[23] E. Aprile et al., Nucl. Phys. Proc. Suppl. 138, 156 (2005), arXiv:astro-ph/0407575, P. L. Brink et al. [CDMS-II Collaboration], In the Proceedings of 22nd Texas Symposium on Relativistic Astrophysics at Stanford University, Stanford, California, 13-17 Dec 2004, pp 2529, arXiv:astro-ph/0503583.

[24] O. Adriani et al. [PAMELA Collaboration], arXiv: 0810.4995; O. Adriani et al., Phys. Rev. Lett. 102, 051101 (2009).

[25] J. Chang et al., Nature 456, 362 (2008).

[26] F. Aharonian et al. [H.E.S.S. Collaboration], Phys. Rev. Lett. 101, 261104 (2008), arXiv:0811.3894.

[27] A. A. Abdo et al. [The Fermi LAT Collaboration], Phys. Rev. Lett. 102, 181101 (2009), arXiv:0905.0025.

[28] W. Buchmuller, L. Covi, K. Hamaguchi, A. Ibarra and T. Yanagida, JHEP 0703, 037 (2007); C. R. Chen, F. Takahashi and T. T. Yanagida, Phys. Lett. B 671, 71 (2009); A. Ibarra and D. Tran, arXiv:0811.1555 E. Nardi, F. Sannino and A. Strumia, JCAP 0901, 043 (2009).

[29] A. Arvanitaki, S. Dimopoulos, S. Dubovsky, P. W. Graham, R. Harnik and S. Rajendran, arXiv:0812.2075

[30] A. Arvanitaki, S. Dimopoulos, S. Dubovsky, P. W. Graham, R. Harnik and S. Rajendran, arXiv:0904.2789.

[31] P. Meade, M. Papucci, A. Strumia and T. Volansky, arXiv:0905.0480.

[32] G. Bertone, M. Cirelli, A. Strumia and M. Taoso, arXiv:0811.3744.

[33] G. Huetsi, A. Hektor and M. Raidal, arXiv:0906.4550

[34] M. Cirelli, M. Kadastik, M. Raidal and A. Strumia, Nucl. Phys. B 813, 1 (2009), arXiv:0809.2409

[35] F. Donato, D. Maurin, P. Brun, T. Delahaye and P. Salati, Phys. Rev. Lett. 102, 071301 (2009) arXiv:0810.5292 [astro-ph]]. 